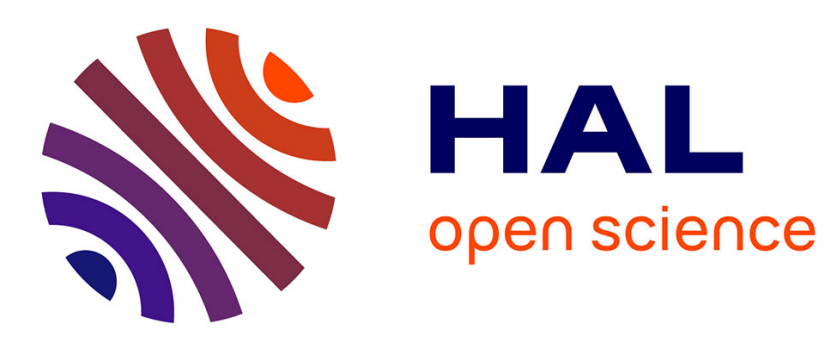

\title{
Discipline et familiarisation à travers la médecine : une mission médicale à Gaza (1878-1814)
}

Philippe Bourmaud

\section{To cite this version:}

Philippe Bourmaud. Discipline et familiarisation à travers la médecine: une mission médicale à Gaza (1878-1814). Histoire et Missions Chrétiennes, 2012, 21, pp.81-102. halshs-00734937

\section{HAL Id: halshs-00734937 \\ https://shs.hal.science/halshs-00734937}

Submitted on 10 Nov 2013

HAL is a multi-disciplinary open access archive for the deposit and dissemination of scientific research documents, whether they are published or not. The documents may come from teaching and research institutions in France or abroad, or from public or private research centers.
L'archive ouverte pluridisciplinaire $\mathbf{H A L}$, est destinée au dépôt et à la diffusion de documents scientifiques de niveau recherche, publiés ou non, émanant des établissements d'enseignement et de recherche français ou étrangers, des laboratoires publics ou privés. 


\section{Philippe Bourmaud, Université Lyon 3 / LARHRA}

\section{Discipline et familiarisation à travers la médecine : une mission médicale à Gaza (1878-1914)}

\section{Introduction :}

Quels buts poursuit une mission médicale ? Cette dernière est par définition équivoque : en tant que mission, elle participe d'une dynamique de présence religieuse, souvent tournée vers la présentation de la foi et l'invitation à adopter celle-ci. Comme institution médicale, elle vise le soin des corps. Entre l'un et l'autre objectif, la relation est dialectique : l'activité thérapeutique peut être envisagée comme une fin en soi, comme une forme de témoignage des croyants, ou comme un moyen d'accéder indirectement au for intérieur de leurs patients. Toute une gradation existe de la médecine au prosélytisme.

Cette ambiguïté est redoublée dans le cas des missions médicales chrétiennes dans le monde musulman: de longue date, ce dernier est considéré comme un champ missionnaire particulièrement difficile, en raison du poids de la construction mutuelle de l'islam et du christianisme comme erreurs religieuses. L'efficacité des missions médicales chrétiennes se mesuret-elle alors à l'aune du nombre des convertis, ou du nombre des patients, ou peut-être de critères plus fins d'évaluation de l'incidence du travail missionnaire?

Parmi les missions médicales que la Church Missionary Society (CMS) de l'Eglise d'Angleterre a fondée en Palestine à partir des années 1870, celle de Gaza constitue un objet intéressant parce qu'elle est décrite à travers les archives comme un succès et un modèle. Et derechef, se pose la question de l'efficacité : succès selon quels criètres, et modèle en quel sens ? Du point de vue du prosélytisme, les résultats sont maigres ; et d'un point de vue médical, les données chiffrées de fréquentation et d'hospitalisation sont à prendre avec un grain de sel, car l'anthropologie et l'histoire de la médecine ont, dans les trente dernières années, montré combien superficiels étaient les effets sanitaires des institutions sanitaires occidentales, missionnaires et autres, d'un point de vue de santé publique ${ }^{1}$. Bien plutôt, il s'agit d'un succès d'influence, spécialement évident en comparaison d'autres missions de la CMS en Palestine, et fondé sur un important travail de socialisation et d'apprentissage linguistique de la part des responsables britanniques de la mission.

1 Roy MacLeod, «Introduction », in Roy MacLeod et Milton Lewis (éds.), Disease,Medicine and Empire. Perspectives on Western medicine and the experience of European expanion, Londres et New York, Routledge, 1988, pp. 1-18. 
Pourtant, il n'est pas possible de faire l'impasse sur le prosélytisme, ou de répudier comme cosmétique l'action médicale des missionnaires ; car tout leur temps est organisé entre les deux pôles de l'évangélisation et de la médecine. Dans le cas de la mission de la CMS à Gaza, la dimension médicale est toujours officiellement subordonnée à la dimension pastorale de la mission; jusqu'à produire un discours sur les besoins spirituels et les besoins de soins des habitants. Au quotidien cependant, activités évangéliques et activités médicales sont toujours l'objet d'un difficile équilibre. En pratique, les médecins missionnaires, très fortement sollicités par leurs patients, trouvent peu de temps pour porter la bonne parole de la religion.

Equilibrant la mission et la médecine, les missionnaires installés à Gaza font un travail de même nature que celui des autres missions de Palestine. Les qualités personnelles des hommes et des femmes travaillant parmi la population gaziote ne sont pas indifférentes pour rendre compte de leur popularité; mais du fait du renouvellement du personnel, surtout infirmier, le facteur personnalité n'explique que partiellement le succès de la mission dans son ensemble. Celui-ci est contre-intuitif, et d'abord parce que la médecine pratiquée par les missionnaires, en salle de consultation et plus encore à l'hôpital, est productrice d'ordre et de disciplines. On connaît, depuis les écrits de Michel Foucault, l'importance des dispositifs entraînant une disciplinarisation des personnes dans les grandes institutions collectifs, l'hôpital comme l'armée ou la prison ${ }^{2}$. C'est donc en dépit de ces dispositifs incommodes, angoissants, contraignants et disciplinants qu'il faut penser le succès de la mission de Gaza ; ou bien il faut supposer que la mise en place desdits dispositifs non seulement répondait tout de même à des attentes de la population gaziote, mais était assez souple pour ne pas rebutter les patients.

L'histoire de la mission médicale de Gaza suggère, à côté des dispositifs disciplinaires hospitaliers, un travail d'apprentissage socio-culturel de la part du personnel missionnaire qui a permis d'humaniser quelque peu ces dispositifs. Une hypothèse, suggérée par certains protagonistes du développement de la mission médicale de Gaza, est que cette capacité de souplesse et de relations étroites résultait de l'organisation de la congrégation protestante locale. Cette dernière aurait servi, non de frein à la socialisation avec les patients et leur entourage, mais au contraire de liant et d'instrument pour désarmer les lourdes idées préconçues des missionnaires à l'égard de la population musulmane de Gaza, et réciproquement. Il reste à voir comment cela a pu se faire.

\section{A quoi tient le « succès » de la mission médicale de la CMS à Gaza ?}

2 Michel Foucault, Surveiller et Punir, Paris, Gallimard, 1975, notamment p. 246-247. 
La mission médicale de Gaza de la CMS est mise en place dans la lignée d'une série de fondation de même type, à Jérusalem, Jaffa et Nazareth ; mais elle s'inscrit dans une inspiration missiologique nouvelle, tournée désormais non vers les communautés chrétiennes orientales, et davantage vers la population musulmane. Ce seul fait redéfinit les attentes des missionnaires : comme pour toutes les missions chrétiennes du Proche-Orient, le prosélytisme en direction des musulmans ne relève pas du possible, ou du moins ne saurait être mené ouvertement, sauf à encourir les foudres de la loi ottomane et la colère des musulmans des environs ${ }^{3}$. La mission médicale est un pari : celui selon lequel il est possible d'exercer une influence et de s'en servir pour faciliter l'évangélisation.

Gaza présente les caractéristiques privilégiées par les missionnaires protestants dans le dernier quart du dix-neuvième siècle. En premier lieu, elle couvre une aire éloignée des premiers foyers de mission de la région, installés dans les années 1820-1840 à Jérusalem et aux alentours, à Nazareth, et dans la montagne libanaise - c'est-à-dire aux Saints-lieux et dans les régions de concentration démographiques des chrétiens orientaux. Fondée par Pritchard, un missionnaire anglican, en 1872, la mission de Gaza dispose à ses début d'un avantage géographique : c'est alors la seule mission protestante de la région, entre Jaffa et Port-Saïd ${ }^{4}$. De manière générale, à la fin du dix-neuvième siècle, l'activité missionnaire étend au Proche-Orient un maillage systématique d'installation de congrégations protestantes, et c'est alors que les missions protestantes à Naplouse, Hébron, Salt et Kérak se développent; localités comparables, par leur enclavement et leur démographie confessionnelle, à Gaza.

\section{Une ville de mission aux caractéristiques standard pour la fin du dix-neuvième siècle}

Car, en deuxième lieu, la ville possède en outre une petite communauté chrétienne au milieu d'une écrasante majorité musulmane. Sous réserve de ce que valent les statistiques démographiques, souvent très trompeuses, données par les voyageurs et résidents européens dans l'Empire ottoman, 1500 des environ 30000 habitants de Gaza, soit environ $5 \%$, sont chrétiens, principalement grecsorthodoxes ${ }^{5}$. D'une manière générale, les chrétientés orientales sont la cible privilégiée des missions protestantes, tant les conversions sont rares à partir du judaïsme, et plus encore de l'islam. Des très nombreuses sociétés missionnaires actives en Palestine, la CMS est de celles qui ont le plus la

3 Sur ce point, et pour les missions catholiques, voir notamment : Jérôme Bocquet, Missionnaires Français en terre d'islam. Damas 1860-1914, Paris, Editions Les Indes savantes, 2005, p. 242 et 259-266.

4 Robert Sterling, « Gaza medical mission », in Medical Mission quarterly, vol. 1, n 4, octobre 1893, p. 10.

5 Robert Elliott, «Adress by Rev. Robert Elliott, L.R.C.S.I., at the C.M.S. Medical missionary meating, May $18^{\text {th }}$, 1894 », in Medical Mission quarterly, vol. 1, n 7 , juillet 1894, p. 8. 
réputation de faire fond sur les chrétiens du Proche-Orient pour obtenir des conversions et fonder des congrégations. Forçant peut-être le trait, l'évêque anglican George Popham Blyth, en poste à Jérusalem à partir de 1887, bientôt en conflit ouvert avec la mission de la CMS en Palestine se plaint ainsi que les habitants du pays ait à l'époque surnommé cette dernière société « les chacals », du fait de leur prosélytisme agressif vis-à-vis d'Eglises locales réputées moribondes ${ }^{6}$. A mesure que les missions protestantes s'éloignent des principaux centres de peuplement chrétien, la CMS s'implante dans des régions essentiellement peuplées de musulmans, mais en s'appuyant d'abord sur chrétiens locaux.

En outre, comme il arrive souvent au Proche-Orient à partir des années 1830, la concurrence religieuse s'installe rapidement entre missions catholiques et protestantes, et cette concurrence de deux confessions nouvelles venues dans la région est souvent accueillie avec scepticisme, voire avec hostilité par les populations, aussi bien chrétiennes que musulmanes ${ }^{7}$. La mission de Pritchard, conduite de façon autonome par son fondateur, s'essouffle rapidement; mais en 1878, un juif converti au protestantisme, Alexander Wilhelm Schapira (1847-1915) ${ }^{8}$ prend sa succession au nom de la CMS et lui donne un nouvel élan. Dès 1879, un missionnaire catholique autrichien, Georg Gatt (1843-1924), s'installe à son tour à Gaza avec l'assentiment du patriarche latin de Jérusalem, Mgr Vicente Bracco, et y fonde une mission et une école ${ }^{9}$.

$\mathrm{Vu}$ de loin, Gaza ne semble donc pas se distinguer des autres missions de la CMS en Palestine ; et son évolution, plus précoce de quelques années que les missions déjà évoquées de la CMS Kérak, Salt et Naplouse, suit une marche standard. L'idée première de l'organisation de la mission de la CMS en Palestine est de fait, dans les premiers temps de l'installation de Schapira, d'inciter ce dernier à concentrer son action sur les Grecs-orthodoxes ${ }^{10}$. Ce dernier y monte en 1879 un petit dispensaire ${ }^{11}$. Il y fait recruter un médecin issu de la faculté de médecine américaine de Beyrouth en août $1881^{12}$; puis un médecin anglais, le Révérend Dr Robert Elliott, en $1886^{13}$. Un

6 Archives Jerusalem \& the East Mission Society (ci-après JEMS), Middle East Centre, St Antony's College, Université d'Oxford, 22/5 : « Notes on the C.M.S. », dactylographiées [1959].

7 Ussama Makdisi, «Corrupting the sublime sultanate : the revolt of Tanyus Shahin in Nineteenth-century Ottoman Lebanon », in Comparative Studies in society and history, vol. 42, $\mathrm{n}^{\circ}$ 1, janvier 2000, p. 180-208.

8 Schapira, à en croire un site australien de généalogie, est originaire de Jaffa, et se convertit au christianisme à l'âge de 26 ans. Il est ensuite ordonné prêtre puis part en mission à Londres, avant de rentrer s'installer comme missionnaire à Gaza, puis Haïfa ; il est par la suite également missionnaire au Sierra Leone, en Tasmanie et en Australie, avant de finir sa vie à Sydney. Informations disponibles en ligne à :

http://www.tasmaniangenealogy.com.au/surnames_s-z.htm, consulté le 15 novembre 2011.

9 Pierre Médebielle, s.j., Gaza et son Histoire chrétienne, Jérusalem, Imprimerie du Patriarcat latin, 1982, p. 70-71.

10 Archives de la Church Missionary Society (Bibliothèque de l'Université de Birmingham; ci-après, CMS), C MO (Mission méditerranéenne) 2 1878/39, « Minutes of a Conference of Missionaries of the Church Missionary Society in Palestine held in Jerusalem March $26^{\text {th }}$ to $28^{\text {th }} 1878 »$.

11 CMS, C MO 64/23, de Schapira à Wright, de Gaza, 19/02/1879; CMS, C MO 2 1880/4, « Minutes of the Seventh Conference of the missionaries of the Church Missionary Society in Palestine held in Jerusalem December 9 th- $12^{\text {th }}$ $1879 »$.

12 CMS, G 3 (Mission de Méditerranée) PO (originaux des papiers personnels) 1844/32, du Dr Asaad Sélim, médecin de la mission de Gaza, au Secrétaire de la CMS, d'Istanbul, 04/02/1884. 
hôpital provisoire est installé en 1892 dans une maison de la ville ${ }^{14}$, avant qu'un vaste hôpital ne soit édifié et finalement ouvert le ler avril $1908^{15}$.

\section{Médecine et priorités missionnaires : la confrontation entre l'évêque Blyth et la CMS}

La station de Gaza s'inscrit bien dans des tendances lourdes des missions protestantes, et en particulie de celles de la CMS. C'est la superposition d'un questionnement missiologique et d'une nouvelle configuration de l'anglicanisme en Palestine qui aboutit à l'essor des missions médicales à partir des années 1880 : l'heure est à une action d'influence, moins agressive au premier abord, et empreinte de considérations caritatives. La médecine est un des piliers de ce mode opératoire missionnaire. Dans le même temps, les missions anglicanes installées en Palestine et au ProcheOrient se tournent bien davantage que naguère vers les musulmans, délaissant relativement les chrétiens orientaux.

Cette réorientation est rendue nécessaire par la nouvelle configuration du protestantisme en Palestine : un protestantisme divisé, essentiellement entre anglicans et luthériens, et dans le cas des Anglicans, soumis à des tensions reproduisant les clivages internes à l'Eglise d'Angleterre. La mission protestante puis anglicane de Palestine connaît un profond changement de dominance théologique au cours des années 1880. Elle était jusque là contrôlée par les tendances évangéliques et protestantes radicales au sein de l'Eglise d'Angleterre, à l'exemple de la CMS. Cette dominance tenait aux conditions originelles de la mission protestante en Palestine, initiée par des millénaristes anglicans et luthériens unis sous un même évêque ${ }^{16}$. A partir de 1885, les Anglicans font cavalier seul $^{17}$; et lorsque Londres nomme un évêque anglican à Jérusalem en 1887, c'est un représentant de la Haute Eglise, Blyth, qui est envoyé. D'emblée, ses relations avec la CMS locale sont mauvaises.

En apparence, c'est une question théologique. Blyth est accusé d'être un « ritualiste » par les missionnaires de la CMS. Il accuse en tout état de cause ces derniers d'éviter soigneusement les services religieux dans les églises du diocèse réputées proches de l'évêque, de peur de perdre le soutien financier des donateurs britanniques les plus stricts sur le plan théologique ${ }^{18}$. Derrière ce premier niveau de lecture du conflit, il y a bien davantage un enjeu d'organisation des congrégations protestantes, c'est-à-dire de priorités missionnaires. Si Blyth réprouve le prosélytisme de la CMS aux dépens des Eglises orientales, c'est que lui-même, ancien missionnaire en Inde, est favorable à

13 «C.M.S. Medical missions. A comparative survey. 10. Gaza, Palestine », in Mercy and Truth, n 200, août 1913, p. 280.

14 R. Sterling, Art. cit., p. 10.

15 C.A. Newton, «A red-letter day at Gaza », in Mercy and Truth, ${ }^{\circ}$ 139, juillet 1908, p. 207.

16 R.W. Greaves, «The Jerusalem Bishopric, $1841 »$, in English Historical review, vol .64, n 252, juillet 1949, p. 328352.

17 Ibid., p. 352.

18 JEMS, 22/5, « Notes on the C.M.S. » [1959]. 
une action missionnaire en direction des majorités religieuses locales - c'est-à-dire des musulmans en Palestine ${ }^{19}$.

Deux visions de la mission s'affrontent donc en Palestine, et compliquent les relations au sein du milieu missionnaire anglican. La CMS est sous pression de Blyth pour favoriser des activités de nature à atteindre les musulmans : l'assistance personnalisée aux femmes, les écoles, les tournées évangéliques itinérantes et, plus encore, la mission médicale. Les tensions au sein du monde anglican de Palestine constituent un facteur important de l'attention accrue accordée par la CMS à la médecine, à la fin des années 1880. Que la mission de Gaza ait été un précurseur sur ce terrain est significatif de ce qui singularise cette mission par rapport aux autres sites d'opération de la CMS.

Gaza, une mission médicale indépendante de l'influence d'une congrégation indigène

Car la mission de Gaza présente une singularité : depuis l'arrivée de Schapira, qui s'est fait de la mission en direction des musulmans une spécialité, elle n'a pas développé de congrégation indigène à partir de convertis venus du christianisme orthodoxe. Elle n'est pas adossée à une congrégation forte, ni surtout à une congrégation de chrétiens orientaux. Ce fait a trois conséquences. D'abord, la congrégation est peu nombreuse : une vingtaine de personnes, mais très visibles d'après un historien du Patriarcat latin ${ }^{20}$. Les membres de la congrégation réunie autour de la mission sont généralement venus d'ailleurs, et sont en fait des musulmans convertis, en rupture de banc avec leur société d'origine : ainsi de deux convertis par le Dr Elliott, médecin missionnaire, le 12 octobre 1890. Marjan et Mabroukah, un couple de Soudanais, avaient été vendus au marché aux esclaves de Gaza avant d'entrer au service domestique du Dr Elliott ${ }^{21}$. Le choix du protestantisme apparaît comme une intégration sociale, à un groupe il est vrai très restreint, pour deux migrants forcés placés en situation d'infériorité sociale et statutaire.

En deuxième lieu, la mission de Gaza est épargnée par les conflits entre les instances locales de la CMS et Blyth. Ce dernier encense le choix précoce qui y a été fait de développer la mission médicale. Il décrit les succès obtenus par le Révérend Dr Sterling en des termes superlatifs («probablement une des missions les plus efficaces au monde ») qui sont sans commune mesure avec le nombre de convertis au protestantisme ${ }^{22}$.

19 Ibid.

20 Médebielle, Op. cit., p. 72.

21 Thomas Edward Dowling, Gaza. A City of many battles (from the family of Noah to the present day), Londres, Society for Promoting Christian Knowledge, 1913, p. 91.

22 Jerusalem and the East Mission Fund (Lambeth Palace, Londres ; ci-après JEMF), 2232, Papiers personnels de Blyth : « Notes made by Bishop Blyth for trustees of the the bishopric, dictated and not signed : date August 29, $1914 »$, Londres. 
En outre, la mission médicale de Gaza, qui emploie les convertis venus de l'islam, est desservie par un personnel indigène moins réticent à précher l'Evangile à des patients musulmans. Dans les villes où les missions sont adossées à des congrégations composées de chrétiens venus d'autres confessions, ces derniers jouent en effet généralement un rôle de frein à l'action missionnaire. Pour Blyth, le « succès » de Gaza tient autant à ce choix de priorité missionnaire qu'à la personnalité flamboyante du nouveau médecin de la mission à partir de 1893, le Révérend Dr Robert Sterling. Que vaut cette interprétation?

Ce qui singularise ce dernier parmi les médecins missionnaires de la CMS en Palestine, c'est en tout cas sa capacité à rallier les suffrages de la population musulmane de Gaza, y compris ses élites, à son travail. Pour le reste, les résultats ne tranchent guère : les conversions sont rares, comme ailleurs. La charge de travail est intense, mais c'est là encore le cas général des dispensaires et hôpitaux missionnaires de Palestine. En revanche, le travail d'acculturation et de relations publiques effectué par Sterling et le personnel de la mission autour de lui est payant. Là où son collègue à Naplouse, le Dr Wright, se discrédite durablement lorsqu'une publication missionnaire révèle ouvertement son dédain des populations indigènes, Sterling, de son côté, est assez habile et populaire pour obtenir que les notables musulmans de la ville apportent en 1906 une contribution financière de $100 £$ aux travaux du nouvel hôpital ${ }^{23}$. Obtenir que la notabilité musulmane finance une institution missionnaire est, pour la région et pour l'époque, inespéré.

On peut accepter l'idée que le personnel indigène, dépris des réticences des chrétiens d'Orient vis-à-vis du prosélytisme chrétien envers les musulmans, se montre plus souple et plus proche des patients. On peut également admettre que, dans le contexte d'une mission résolument tournée vers la majorité musulmane de la population, les missionnaires, surtout européens, fassent un effort supplémentaire d'acculturation sur le plan social et linguistique. La participation des notables musulmans de Gaza au financement de l'hôpital de la CMS est une preuve majeure de la capacité de la mission à désarmer les oppositions politico-religieuses qu'elle avait initialement suscitées, et le malaise, sinon les résistances culturelles, qu'elle pouvait instiller chez les patients et dans leur entourage ; mais est-ce qu'un tel travail en direction de la population gaziote n'est pas d'emblée contré par le caractère disciplinaire des institutions médicales ?

\section{L'ambivalence de la discipline}

La mission médicale de Gaza est un lieu de disciplinarisation, de contrôle des conduites

23 Dowling, Op. cit., p. 91. 
individuelles non par exercice d'un pouvoir humain mais par les contraintes d'un dispositif ; mais la discipline n'y répond pas exclusivement aux attentes des missionnaires britanniques vis-à-vis de la population indigène, elle est réversible car, dans l'histoire de la mission, les exigences de contrôle social s'appliquent également au personnel de la mission.

\section{Organiser pour normer?}

Il ne s'agit pas de nier la dimension disciplinaire des institutions médicales et surtout hospitalière, à Gaza pas plus qu'ailleurs. La structure architecturale de l'hôpital missionnaire ouvert en 1908, n'est pas panoptique, mais plutôt monobloc. Son organisation interne vise à instaurer ou à reproduire des codes moraux. L'étage supérieur est réservé aux patientes ${ }^{24}$, ce qui est une caractéristique générale des hôpitaux missionnaires de l'époque en Palestine : c'est le moyen d'éviter que ces dernières ne soient dans une partie passante de l'établissement, ou ne puissent être vues de l'extérieur. De même, les salles de malades de l'hôpital sont organisées dortoirs, avec quelques chambres plus petites pour la clientèle privée ${ }^{25}$; un système qui facilite l'examen clinique quotidien, mais peut être également conçu dans une visée de surveillance - la surveillance mutuelle entre les patients.

Cette organisation répond à des normes hospitalières européennes, mais elle contribue aussi à respectabiliser l'hôpital, en le concevant de façon à répondre à ce qui est perçu comme autant de normes autochtones sur les rapports de genre ou la différence entre la sphère privée et la sphère publique : l'hôpital, lieu public et lieu d'hébergement, ne doit pas devenir un lieu d'immoralité, et cette préoccupation est prégnante dans les différentes missions de la CMS en Palestine. Cette exigence est disciplinante aussi bien pour les missionnaires que pour les patients.

Un bon exemple, ici, est l'accueil des patients et des patientes en clinique ambulatoire. A la différence d'autres missions en Palestine, la mission médicale de Gaza n'a pas instauré un système de journées différenciées : journée des hommes, journée des femmes et des enfants ${ }^{26}$. Le voudraiton que ce ne serait pas possible, du fait de l'arrivée chaque jour de patients venus de très loin pour consulter $^{27}$.

L'organisation de la clinique ambulatoire est l'occasion d'un sérieux conflit interne fin 1894, entre le Dr Sterling, débordé de tavail depuis son arrivée deux ans plus tôt, et ses deux infirmières

24 Newton, Art. cit., p. 207.

25 Ibid., p. 207-209.

26 CMS G 3 P/O 1895/54, de Hall à Baylis, de Jérusalem, 14/03/1895.

27 Sterling, Art. cit., p. 11. 
britanniques, Miss Campbell et Miss Patching. Ces dernières se plaignent que le médecin laisse homme et femmes venir ensemble en consultation. La salle de consultation étant ce qu'elle est - un couloir sans salle d'attente - les patients qui attendent peuvent voir les examens en train d'avoir lieu. Le secrétaire de la mission de Palestine, Hall, intervient aussitôt pour rappeler Sterling à l'ordre : si, pour de petites opérations, il n'y a pas de mal à accueillir hommes et femmes ensemble dans la salle de consultation, c'est inacceptable dès lors d'un examen corporel, même le plus limité. Ce serait une cause de scandale dans un pays musulman, et considéré à la limite de l'immoralité. Sterling donne des ordres au portier pour interdire l'accès de la salle de consultation aux hommes et aux femmes au même moment. Pourtant, les infirmières réitèrent leurs plaintes; et le Dr Sterling d'expliquer que malgré ses ordres, il arrive qu'un patient soit laissé à attendre dans la salle de consultation pour un examen approfondi, et qu'en attendant, une patiente soit admise dans la série des patients suivants. Sterling admet que, durant les consultations, il est tellement absorbé par son travail, qu'il oublie entièrement la présence de ceux qu'il a fait attendre plus tôt dans la journée ${ }^{28}$.

L'affaire, révélant des incompatibilités de personnes au sein du personnel missionnaire, aboutit à la démission de Miss Campbell ${ }^{29}$; mais elle montre également un travail progressif de conception des règles de la mission médicale dans un effort pour se conformer à des normes autochtones; et la difficulté, pour les missionnaires, d'organiser leur travail pour se plier à ces mêmes normes.

Une quinzaine d'années plus tard, en 1911, la question de l'organisation de l'accès à la consultation ressurgit, lorsqu'est ouvert le nouveau bâtiment de la clinique ambulatoire dans le complexe de l'hôpital. A cette date, la mission médicale dispose de plus de personnel pour assurer la surveillance ; et le bâtiment comporte désormais deux salles de consultation, auxquelles on accède par un couloir tracé par des barrières, et terminé par un tourniquet. Le dispositif d'accès a cette fois été conçu pour discipliner les entrants, à commencer par la division de la salle de consultation pour séparer les deux sexes ${ }^{30}$. Même si les visiteurs admirent la modernité du système, comparé avec quelque emphase aux grandes gares européennes du temps, son inspiration n'est nullement exceptionnelle : dans toutes les missions britanniques en Palestine, suivre l'ordre de passage en consultation est l'objet d'une lutte permanente : ici un tourniquet, ailleurs un système de tickets de passage, classés par ordre d'arrivée ou contingentés ${ }^{31}$. Il ne s'agit pas seulement, ici, de corriger des conduites inappropriées de la part des patients, comme l'habitude de jouer des coudes ou le voyeurisme : le portier et la peur du scandale y suffiraient. Il faut aussi créer un ordre collectif, qui ne résulte pas de la contrainte sur les comportements individuels, mais de leur canalisation : cette

28 CMS G 3 P/O 1895/54 : Hall à Baylis, Jérusaalem, 14/03/1895. Annotée « Private \& confidential. »

29 Ibid.

30 Henry Sykes, «New Accommodation for out-patients at Gaza hospital », in Mercy and Truth, $\mathrm{n}^{\circ}$ 174, juin 1911, p. 188.

31 CMS G 3 P/O 1892/7, de Bailey à Zeller, de Naplouse, 11/12/1891. 
forme si culturellement située qui s'appelle la file d'attente. Sous-jacente est l'idée culturaliste qu'il ne peut, au Proche-Orient, y avoir d'auto-discipline.

Cette pensée n'est sans doute pas absente de l'esprit des concepteurs de l'hôpital de 1908 ; mais, mis en perspective avec les incidents de 1894, les dispositifs disciplinaires qui ordonnent le nouvel édifice sont irréductibles à ce culturalisme, car les patients indigènes ne sont pas leur unique point d'application. L'effet d'ordre induit par le tourniquet et la séparation des files touche aussi bien les visiteurs de l'hôpital que son personnel, Britanniques inclus.

\section{Discipliner pour prêcher}

Ce qui vaut de l'organisation du travail médical vaut également de l'activité missionnaire. Celle-ci est, à Gaza comme dans les autres missions médicales, organisée de façon très normative. Elle est rythmée par les séances de prière et les adresses religieuses aux présents, suivi des soins : le matin pour la clinique ambulatoire, l'après-midi dans les salles de malades. Seuls les patients qui ont assisté au service préalable à la consultation sont admis à cette dernière, conformément aux instructions de la CMS de subordonner le travail médical à l'évangélisation.

Les descriptions que les missionnaires donnent de ces services religieux, à l'attention de la maison-mère, sont assez lénifiantes : une assistance intéressée, de la bienveillance de la part des patients pour le volet proprement missionnaire de la mission médicale. Mais entre les lignes, une autre réalité se dégage : celle de patients qui s'assoupissent, quitte à être réveillé par le médecin levantin de la mission ; ou de «Yistaghfir Allah!» (A Dieu ne plaise!) murmurés lorsque sont abordés les points de la doctrine chrétienne les plus contestés en islam, comme la mort et la résurrection de Jésus ${ }^{32}$. Autrement dit, le principalement moment de prosélytisme dans le quotidien de la mission médicale est accueilli avec une indifférence policée de gens qui, venus se faire soigner, entendent traiter les missionnaires avec respect, sans plus ; à telle enseigne que, pour sortir l'assistance de cette indifférence, le Dr Sterling affirme parfois lancer la discussion à la cantonnade, sur les sujets théologiques conflictuels ${ }^{33}$.

En définitive, l'attitude des missionnaires ne fait pas scandale, à la différence d'autres villes de Palestine. A Gaza, les relations restent polies ; et si la mission médicale est fortement décriée à ses débuts, c'est pour des raisons de politique locale, inhérentes aux rivalités entre grandes familles gaziotes. Entre celles-ci, qui forment des factions rivales pour le pouvoir local - et qui, au désespoir de l'historien, ne sont jamais nommément citées par les missionnaires -, on s'échange, dans les 
premiers temps du service de Sterling, les accusations de complaisance à l'égard de missionnaires ${ }^{34}$. De fait, ces derniers recherchent activement la sympathie des notables, alors qu'ils sont vus comme les fourriers de la colonisation : ce genre de soutien est une sécurité pour la pérennité de leur travail évangélique. Un tel travail d'approche des officiels ottomans et des notabilités locales n'est nullement spécifique de la mission médicale de Gaza : à peu près tous les médecins missionnaires de la CMS soignent gratuitement, à titre de faveur, le gouverneur local ou le mufti de leur ville de résidence dans les premiers temps de leur installation. Cependant, à Gaza, cette obligation de socialisation se conjugue à l'absence d'une congrégation protestante locale légitime aux yeux des autorités (c'est-à-dire issue des chrétientés orientales, et non de l'islam à partir duquel la conversion est interdite). Là où les chrétiens locaux convertis au protestantisme sont présents, ils servent souvent d'intermédiaires avec les autorités, et souvent d'interprètes. A Gaza, en l'absence de tels relais, le personnel de la CMS s'astreint visiblement plus qu'ailleurs à un travail rigoureux d'apprentissage des outils culturels et linguistiques de communication ; travail qui, du reste, fait partie de leurs obligations en vue de leur activité proprement missionnaire.

\section{Les apprentissages croisés d'un système d'interactions médicales}

\section{Un douloureux apprentissage linguistique}

L'apprentissage de l'arabe, qui met à rude épreuve tous les missionnaires médicaux, est posé par la mission comme une double obligation : obligation pastorale et obligation médicale.

C'est une obligation pastorale, parce que la mission est toujours pensée comme prioritairement motivée par l'objectif de la conversion. Et qu'il faut donc pouvoir non seulement prêcher en arabe et chanter les hymnes en arabes, mais également s'entretenir individuellement en arabe avec les patients sur les questions théologiques et spirituelles.

Egalement, c'est une obligation médicale, pour comprendre et interpréter ce que disent les patients, et qui n'est en rien transparent.

La question linguistique tend à structurer la mission. Elle pose une alternative : soit il convient d'embaucher un médecin indigène, par conséquent arabophone ; soit, si l'on fait venir un médecin européen, il faut lui imposer un apprentissage initial intensif de la langue, tout en le faisant travailler dans un premier temps avec un traducteur.

Aucune de ces deux options n'est pleinement satisfaisante pour la société missionnaire.

A Gaza, on a dans un premier temps recours à un médecin protestant originaire de la montagne libanaise ; mais presque aussitôt, le révérend Schapira - dont nous avons vu qu'il prenait 
ses distances avec les chrétiens orientaux convertis au protestantisme - demande que lui soit substitué un médecin européen ${ }^{35}$. Le consensus à la CMS est qu'il est virtuellement impossible de trouver un homme, parmi les rares médecins protestants du Proche-Orient, qui ait aussi une réelle vocation missionnaire $^{36}$. Et ce, même si certains visiteurs reconnaissent l'avantage que leur confère leur plus grande familiarité culturelle et linguistique avec les patients ${ }^{37}$.

Après le renvoi de ce premier médecin pour des raisons éthiques ${ }^{38}$, la CMS s'arrange pour toujours nommer un médecin-chef européen ; quitte à trouver expédient d'embaucher, après quelques années, un médecin proche-oriental pour l'assister. Dès lors, le difficile apprentissage de l'arabe devient le lot de tous les missionnaires britanniques à Gaza, y compris pour des missionnaires madrés, anciens des missions médicales sous diverses latitudes : ainsi du Dr Elliott, longtemps missionnaire à Santal, au Bengale ${ }^{39}$.

La première et la plus inhibante difficulté pour acquérir la langue arabe est une question de temps disponible : pendant deux ans, le Dr Sterling ne réussit pas à équilibrer son travail médical écrasant et son activité missionnaire. Il réussit d'autant moins à se libérer pour l'apprentissage linguistique ; mais passé cette phase, ce n'est pas seulement son dynamisme, mais également sa compétence linguistique et la durée de son poste (de 1893 à 1914) qui contribuent à en faire une figure de la vie locale. Il devient un arabisant assez éminent pour publier A Grammar of the Arabic language et Arabic and English Idiom - Conversational and Literary, qui attestent d'une maîtrise des différents registres de cette langue ${ }^{40}$. Comparativement, le Dr Wright, qui occupe les mêmes fonctions à Naplouse durant à peu près les mêmes années, reste une personnalité controversée : ses hésitations en arabe n'y sont pas pour peu ${ }^{41}$.

Miss Watney, qui travaille brièvement à la mission de Gaza comme infirmière en 1896-1897, rend compte d'une variété d'autres difficultés d'apprentissage. Le premier problème est une question d'intelligibilité de la part des patients et des habitants de la ville : en dépit du temps qu'elle consacre à l'étude de l'arabe, ses interlocuteurs répondent avec une insatisfaction constante à ses timides efforts pour parler. En retour, elle se gausse de la prétention des Gaziotes anglophones à parler parfaitement la langue de Chaucer : lesquels restent incrédules lorsqu'elle leur dit que les anglophones de naissance peuvent reconnaître un non-anglophone dès le premier mot.

L'apprentissage de l'arabe possède, à la mission médicale, une dimension immédiatement

35 CMS G 3 P/O 1884/118 : d'A.W. Schapira à Lang, de Gaza, 31/05/1884.

36 CMS G 3 P/O 1891/178 : de Hall à Hamilton, de Hampstead, 02/06/1891.

37 CMS G 3 P/O 1885/36 : du Dr Dowell à Lang, de Gaza, 07/02/1885.

38 CMS G 3 P/O 1885/133 : «Minutes of the eighteenth conference of the Palestine Mission of the Church Missionary Society held in Jerusalem, June $7^{\text {th }}$ to $10^{\text {th }} 1885$. »

39 «C.M.S. Medical missions. A comparative survey. 10. Gaza, Palestine », in Mercy and Truth, n 200, août 1913, p. 280.

40 Dowling, Op. cit., p. 91.

41 CMS G 3 P/O 1904/30 : de Hall à Baylis, de Jaffa, 27/02/1904. 
pragmatique, qui révèle aisément, parfois de façon embarrassantes, des compétences linguistiques limitées. Le journal de Miss Watney qui, destiné aux publications missionnaires, s'efforce de donner dans le pittoresque et l'anecdote légère, insiste non seulement sur ses efforts laborieux, mais également sur le caractère cocasse des circonstances que son niveau d'arabe limité lui donne dans l'entretien avec les patients. S'efforçant d'expliquer à un patient de l'hôpital l'usage d'un thermomètre, elle conseille de le placer sous la langue, de ne pas mordre, et de refermer la bouche. Vainement, car l'homme reste bouche ouverte - jusqu'au moment où elle comprend qu'elle a confondu les verbes $«$ ouvrir $»$ et $\ll$ fermer ${ }^{42}$.

\section{Un réapprentissage du colloque singulier}

En sens inverse, la mission médicale est également un lieu de familiarisation avec la médecine occidentale pour les habitant de la région. D'une part, c'est un lieu de formation professionnelle : la mission médicale abrite en permanence des infirmières autochtones qui y font leurs preuves. D'autre part, et surtout, la consultation est un lieu de traduction séméiologique. Dans la littérature missionnaire, le Dr Sterling insiste sur la démarche systématique de questionnement dans le colloque singulier, et par conséquent sur ses efforts pour amener les patients à formuler leurs sensations et leurs souffrances dans un cadre normatif ; mais ses descriptions montrent en creux son travail linguistique pour traduire dans son propre idiome médical les paroles de ses patients.

Dans les premiers mois de son travail à Gaza, il se plaint de l'imprécision anatomique de la description que les patients font de leurs douleurs. A la question de savoir ce qui ne va pas, on lui répond par exemple «ras kalbi », que Sterling traduit par «la tête de mon coeur»; ou, plus idiomatiquement, «le haut de mon coeur» (ou de ma poitrine), ce qui commence à définir une localisation anatomique. De la même manière, lorsqu'il demande à une mère ce dont souffre son fils, elle élude la réponse en disant que c'est à lui, médecin, de trouver. Puis elle ajoute qu'il a une fièvre - terme indéfini aux yeux de Sterling ${ }^{43}$.

Il n'y a rien ici qui doive surprendre, et le problème de la traduction séméiologique est certes culturel - car il confronte les codes et perceptions du médecin et du profane -, mais il n'est pas pour autant interculturel : il existe aussi entre individus appartenant à une même aire culturelle, à un même milieu socio-culturel. Ainsi que l'explique Aaron Cicourel dans ses travaux sur le raisonnement médical, le colloque singulier est l'occasion de fréquentes méprises entre médecin et patient. Ces malentendus sont conditionnés par les attentes et les habitudes des uns et des autres, ainsi que par la relation médecin-patient comme relation de pouvoir articulée à l'asymétrie du 
savoir $^{44}$.

Dans l'interaction mentionnée ci-dessus, comment interpréter la réponse de la mère, lorsqu'elle renvoie le médecin à sa propre prétention au savoir médical ? Elle est inhabituelle par rapport à ce qu'on peut savoir des normes du colloque singulier dans la médecine traditionnelle ottomane : à savoir que les patients ont, au contraire, une prétention à se connaître et à connaître ce dont ils souffrent. En 1848, deux générations avant l'arrivée du Dr Sterling, un des premiers missionnaires médicaux protestants au Proche-Orient, Cornelius Van Dyck, note que les patients de la montagne libanaise ont toujours une certaine idée de ce dont ils souffrent, et réussissent généralement à l'exprimer et à lui donner sens dans le langage de la théorie humoraliste ${ }^{45}$. Il en reste peut-être quelque chose dans la description de Sterling :

«des patients atteints de maux aigus, dit-il, provoquant des symptômes marqués, ne semblent jamais reconnaître la nature de leur maladie, ou à la relier à l'organe impliqué ; par exemple, un homme émacié atteint de dysenterie chronique raconte une histoire de maux de tête et de fièvre. Ce n'est, pourtant, qu'en posant une série de question que l'information nécessaire est retirée, permettant l'élaboration d'un diagnostic correct, et on trouve alors qu'il s'agit d'un mal ordinaire, dont les symptômes ne sont pas associés dans l'esprit de la mère à la cause de la maladie ${ }^{46}$. »

Cette «histoire de maux de tête et de fièvre » renvoie à une connaissance traditionnelle de ce qui, dans l'ordre des symptômes, est significatif dans le cadre épistémologique de la médecine des humeurs. Pour son lecteur, Sterling euphémise l'importance de cette description, au profit de la recherche d'autres symptômes plus significatifs pour lui, en tant que médecin européen. Par là, il exprime la difficulté des médecins formés à l'occidentale à imposer aux patients proche-orientaux un cours systématique durant l'entretien avec le patient. Lorsque la mère citée plus haut suggère au médecin de faire son travail et de trouver lui-même le mal dont souffre son enfant, c'est au contraire au fondement du raisonnement médical inculqué à l'université - l'asymétrie cognitive entre patient et médecin face à la maladie - qu'elle renvoie de façon ironique. Même de façon distanciée, elle montre l'acculturation progressive du nouveau régime des relations entre médecin et patient.

De manière générale, le guidage du colloque singulier par le médecin commence à s'imposer régionalement dans les années 1890. Sterling n'est pas le seul à y insister. Au même moment, à la faculté catholique de médecine de Beyrouth, tout l'accent est mis sur l'apprentissage, par les futurs médecins, d'un questionnaire systématique à poser aux malades ${ }^{47}$. Après 20 ans d'activité, Sterling,

44 Aaron V. Cicourel, Le Raisonnement médical. Une approche socio-cognitive, Paris, Seuil, 2002, passim.

45 Cornelius Van Dyck, «On the present condition of the medical profession in Syria», in Journal of the American Oriental Society, Vol. 1 (1848-1849), pp. 561-591; reprint in Ghada Yusuf Khoury, The Founding Fathers of the American University of Beirut. Biographies, Beirut, AUB Press, 1992, p. 213.

46 Robert Sterling, «Medical work at Gaza », Medical Mission quarterly, vol. $\mathrm{n}^{\circ}$ 1, $\mathrm{n}^{\circ}$ 8, octobre 1894, p. 5-6.

47 Centre des Archives Diplomatiques de Nantes, Archives des Postes, Consulat de Beyrouth, série A, 184, « Rapport 
quitte la mission sur un constat plutôt inverse, et peut-être triomphaliste : selon lui, la mission médicale a imposé son modus operandi par-dessus les préjugés, y compris les croyances médicales populaires $^{48}$. L'apprentissage du colloque singulier par les patients avait pour condition nécessaire un apprentissage préalable par le médecin, suffisamment poussé pour autoriser ce dernier à retraduire les mots et les présupposés épistémologiques de ses patients dans son propre langage médical.

\section{Conclusion :}

En introduction à ce propos, j'ai décrit le «succès » de la mission médicale comme un « succès d'influence »; une influence qui ne passe pas seulement par la faveur, assez relative, des officiels et des notables ottomans, mais aussi par la conformation des patients à des modèles médicaux.

La littérature missiologique des années 1890-1900 revient souvent sur la dimension pédagogique de la mission médicale, notamment dans l'ordre du propre et du sale - une obsession pour les missionnaires, qui n'est peut-être pas exempte d'un arrière-plan religieux : la propreté n'estelle pas réputée proche de la divinité ? Mais dans les processus que j'ai voulu mettre ici en avant, il s'agit davantage d'une familiarisation et d'ajustements mutuels face à des attentes : attentes en termes de moralité, de codes sociaux, de correction linguistique. Cette pédagogie fonctionne mieux parce que l'effort d'apprentissage de l'arabe consenti par le personnel britannique, et d'abord par le Dr Sterling, est plus manifeste et plus réussi qu'ailleurs. Que Sterling ait un véritable talent d'arabisant est vraisemblable, mais il n'est pas le seul au sein de la mission de Palestine ; c'est la dimension pragmatique de son usage de cette langue qui fait la différence. A ce titre, on peut sans doute valider la thèse de l'évêque Blyth : dépourvue d'une congrégation protestante indigène formée à partir des chrétientés orientales, la mission de Gaza est plus portée que les autres stations de Palestine à se tourner vers la forte majorité musulmane des habitants, et son personnel a dû faire d'emblée un plus grand effort de socialisation. Plus clairement missionnaire en direction des musulmans, le Dr Sterling et ses collègues à Gaza réussissent mieux à rendre acceptable leur activité évangélique qu'ailleurs ; mais dans ce processus, le passage par la médecine comme moyen de briser la glace est fondamental.

Cette interprétation n'explique bien sûr pas tout : on sait combien les facteurs individuels, comme la présence d'un directeur enthousiasmant ou au contraire morose et maladroits peuvent

adressé à Monsieur le Ministre de l'Instruction Publique [Chaumié] par le Docteur L. Hugounenq, Professeur à la Faculté de Médecine de l'Université de Lyon, Président du jury chargé de faire subir leurs examens aux élèves de la

Faculté libre de médecine et de pharmacie de Beyrouth (Syrie) (Session de février 1903) », s.d. [1903].

48 Robert Sterling, « Medical work in a Moslem stronghold », in Mercy and Truth, $\mathrm{n}^{\circ}$ 225, septembre 1915 , p. 316. 
jouer sur le devenir d'une institution. Il reste que le Dr Sterling, par l'évolution de ses relations avec les patients en consultation, rend manifeste l'incidence du travail linguistique collectif des missionnaires de Gaza sur l'activité médicale proprement dite. Moins superficielle que les chiffres de fréquentation, l'acculturation somme toute rapide du colloque singulier à l'européenne repose sur des relations de compréhension mutuelle. Dès lors, l'environnement de l'hôpital n'est certes pas moins disciplinaire et angoissant, mais ces travers posent moins de problème aux patients qui ont un accès linguistique et affectif plus aisés au personnel que dans les autres missions de la région. 